\title{
Monocyte subsets differentially employ CCR2, CCR5, and CX3CR1 to accumulate within atherosclerotic plaques
}

\author{
Frank Tacke, ${ }^{1}$ David Alvarez, ${ }^{1}$ Theodore J. Kaplan, ${ }^{1}$ Claudia Jakubzick, ${ }^{1}$ Rainer Spanbroek, ${ }^{2}$ \\ Jaime Llodra, ${ }^{1}$ Alexandre Garin, ${ }^{3}$ Jianhua Liu, ${ }^{4}$ Matthias Mack, ${ }^{5}$ Nico van Rooijen, ${ }^{6}$ Sergio A. Lira, ${ }^{3}$ \\ Andreas J. Habenicht, ${ }^{2}$ and Gwendalyn J. Randolph ${ }^{1,3}$

\begin{abstract}
'Department of Gene and Cell Medicine, Mount Sinai School of Medicine, New York, New York, USA. 'Institute for Vascular Medicine, Friedrich-Schiller-University, Jena, Germany. ${ }^{3}$ Department of Immunobiology, Icahn Medical Institute, and ${ }^{4}$ The Recanati/Miller Transplantation Institute, Mount Sinai School of Medicine, New York, New York, USA. 5Department of Internal Medicine, University Clinic, University of Regensburg, Regensburg, Germany. ${ }^{6}$ Department of Molecular Cell Biology, Free University Medical Center, Amsterdam, The Netherlands.
\end{abstract}

\begin{abstract}
Monocytes participate critically in atherosclerosis. There are 2 major subsets expressing different chemokine receptor patterns: $\mathrm{CCR2}{ }^{+} \mathrm{CX} 3 \mathrm{CR} 1^{+} \mathrm{Ly}-{ }^{6} \mathrm{C}^{\mathrm{hi}}$ and CCR2-CX3CR1 ${ }^{++} \mathrm{Ly}-6 \mathrm{C}^{\mathrm{lo}}$ monocytes. Both $\mathrm{C}-\mathrm{C}$ motif chemokine receptor 2 (CCR2) and $\mathrm{C}-\mathrm{X}_{3}-\mathrm{C}$ motif chemokine receptor 1 (CX3CR1) are linked to progression of atherosclerotic plaques. Here, we analyzed mouse monocyte subsets in apoE-deficient mice and traced their differentiation and chemokine receptor usage as they accumulated within atherosclerotic plaques. Blood monocyte counts were elevated in apoE $\mathrm{E}^{-/-}$mice and skewed toward an increased frequency of CCR2 ${ }^{+} \mathrm{Ly}-6 \mathrm{C}^{\mathrm{hi}}$ monocytes in apoE ${ }^{-/-}$mice fed a high-fat diet. CCR2 ${ }^{+} \mathrm{Ly}-6 \mathrm{C}^{\mathrm{hi}}$ monocytes efficiently accumulated in plaques, whereas CCR2Ly- $6 \mathrm{C}^{\text {lo }}$ monocytes entered less frequently but were more prone to developing into plaque cells expressing the dendritic cell-associated marker CD11c, indicating that phagocyte heterogeneity in plaques is linked to distinct types of entering monocytes. CCR2- monocytes did not rely on CX3CR1 to enter plaques. Instead, they were partially dependent upon CCR5, which they selectively upregulated in apoE $\mathrm{E}^{-/-}$mice. By comparison, CCR2 ${ }^{+}$Ly- $6 \mathrm{C}^{\mathrm{hi}}$ monocytes unexpectedly required CX3CR1 in addition to CCR2 and CCR5 to accumulate within plaques. In many other inflammatory settings, these monocytes utilize CCR2, but not CX3CR1, for trafficking. Thus, antagonizing CX3CR1 may be effective therapeutically in ameliorating CCR2 ${ }^{+}$monocyte recruitment to plaques without impairing their CCR2-dependent responses to inflammation overall.
\end{abstract}

\section{Introduction}

Monocytes are critical mediators of atherosclerosis. They traverse the arterial endothelium to enter developing and established lesions, and this trafficking is governed by chemokines. Atherosclerotic lesions are markedly diminished in $\mathrm{apoE}^{-/-}$mice that cannot express the chemokine CCL2 (1) or its receptor C-C motif chemokine receptor 2 (CCR2) (2). CCR1, CCR5, and C-X-C motif chemokine receptor 2 (CXCR2) are other chemokine receptors proposed to affect monocyte recruitment in mouse models of atherosclerosis (3-7). Still another chemokine receptor, CX3CR1, has been linked to atherosclerosis in both mouse models and human studies. Humans who carry a gene encoding the M280 polymorphism in the $\mathrm{C}-\mathrm{X}_{3}-\mathrm{C}$ motif chemokine ligand 1 (CX3CL1) locus that renders the molecule less functional in adhesion studies are relatively protected from cardiovascular disease $(8,9)$, and apoE ${ }^{-/-}$mice lacking CX3CL1 or its receptor $\mathrm{C}-\mathrm{X}_{3}$-C motif chemokine receptor 1 (CX3CR1) have reduced atherosclerosis (10-12). The mechanism(s) explaining the role of CX3CL1 in atherosclerosis are still lacking. While some hypotheses put forth the idea that CX3CL1 may, like CCR2 and others, regulate monocyte recruitment into the plaque $(13,14)$, it is also or alternatively possible that CX3CL1 affects criti-

Nonstandard abbreviations used: CCR2, C-C motif chemokine receptor 2; CXCR2, $\mathrm{C}-\mathrm{X}-\mathrm{C}$ motif chemokine receptor 2; CX3CL1, C-X 3 -C motif chemokine ligand 1; CX3CR1, C-X $\mathrm{X}_{3}-\mathrm{C}$ motif chemokine receptor 1 .

Conflict of interest: The authors have declared that no conflict of interest exists. Citation for this article: J. Clin. Invest. 117:185-194 (2007). doi:10.1172/JCI28549. cal properties of smooth muscle cells, since smooth muscle cells express CX3CL1 and CX3CR1 within lesions (14).

Although CCR2 in particular is considered to be a classical chemokine receptor expressed by monocytes, not all monocytes express CCR2 $(15,16)$. In humans as well as in mice, all monocytes express CX3CR1, but those that express the highest levels of CX3CR1 constitute the nonclassical subset of monocytes that does not express CCR2 $(16,17)$. These monocytes, which in mice are identified by low levels of surface Ly-6C (also called Gr-1) (16), emigrate relatively poorly to sites of acute inflammation, compared with CCR2 ${ }^{+}$classical monocytes $(16,18,19)$. The trafficking patterns of CCR2-CX3CR1 ${ }^{\text {hi }}$ monocytes are so far elusive, and it is unclear whether they would be relevant in atherosclerosis, as they may not be recruited to sites of inflammation such as atherosclerotic plaques. On the other hand, if they do enter plaques, they may very well utilize CX3CR1 to emigrate there.

Given the importance of monocytes and CX3CR1 in atherosclerosis, we set out to address these unknowns. We also assessed whether the heterogeneity of "macrophages" in plaques was related to their origin from the different monocyte subsets. Specifically, some presumed macrophages in plaques express unconventional markers such as CD11c $(20,21)$, which is usually not found on macrophages but instead marks antigen-presenting DCs (22). The CD11 $\mathrm{c}^{+}$cells within atherosclerotic plaques may have important roles in disease, as restoration of their ability to emigrate out of lesions correlates with plaque regression (20). Because the CX3CR1 ${ }^{\text {hi }}$ CCR2- ${ }^{-}$subset of monocytes has been observed to express low levels of CD11c in 

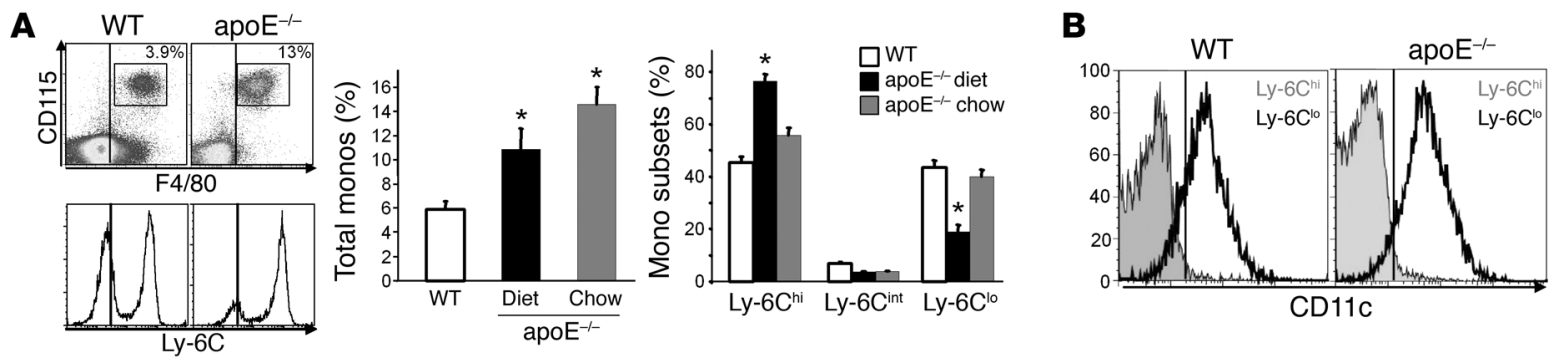

\section{Figure 1}

Characterization of blood monocytes in WT and apoE-/- mice. (A) The frequency of monocytes (monos) was determined in WT mice and in 16-week-old apoE ${ }^{-/-}$mice fed a standard chow (Chow) or 12 weeks of a high-cholesterol/high-fat diet (Diet). Left: dot plots and histograms of stained monocytes; middle panel: bar graph of total monocyte frequency. The relative frequencies of the 2 subsets of blood monocytes (Ly-6Chi and Ly- $6 \mathrm{C}^{10}$ ) and the minor Ly-6C int subset were determined. apoE ${ }^{-/-}$mice kept on a high-fat diet had significantly more Ly-6Chi and fewer Ly-6Clo blood monocytes. Plots show the mean values $( \pm$ SEM) for $12-16$ mice in each condition. *Significantly different from WT, $P<0.001$. (B) Histograms show CD11c expression of either CD115+Ly-6Chi (gray) or CD115+Ly-6Clo cells (black). Plots are representative of flow cytometric analyses for more than 10 animals studied per group. Cut-off for isotype-matched control mAb staining is shown by vertical lines in the plots.

the circulation of mice (18) and may be predisposed to differentiation toward DC-like cells in humans (23), we wondered whether the CD11 $\mathrm{c}^{\text {hi }}$ cells within atherosclerotic plaques of apoE $\mathrm{E}^{-/-}$mice arose from CX3CR $1^{\text {hi }}$ Ly- $6 C^{\text {lo }}$ monocytes rather than CCR2 ${ }^{+}$Ly- $6 \mathrm{C}^{\text {hi }}$ monocytes. Thus, here we used methods recently developed in our laboratory (24) to label and trace the 2 major monocyte subsets in atherosclerotic plaques. We documented their phenotype in apoE ${ }^{-/}$ mouse blood; assessed whether and at what rate each subset entered plaques; traced their differentiation into $\mathrm{CD} 11 \mathrm{c}^{-}$macrophages or $\mathrm{CD} 11 \mathrm{c}^{+} \mathrm{DC}$-like cells within lesions; and compared the role of chemokine receptors - particularly CCR2, CCR5 and CX3CR1 - in mediating their entry into plaques.

\section{Results}

Increased blood monocyte levels in apoE $E^{-/-}$mice. Mouse blood monocytes coexpress CD115 (M-CSF receptor) and F4/80 $(18,19)$. There are 3 subpopulations ( 2 major) of monocytes with distinct chemokine receptor profiles that express high, intermediate, or low/absent levels of Ly-6C (Ly-6Chi ${ }^{\text {Ly- }} 6 \mathrm{C}^{\text {int }}$, and Ly-6C $\mathrm{C}^{\mathrm{lo}}$, respectively) $(16,18,19)$. apoE-/- mice had nearly a doubled frequency of circulating monocytes compared with $\mathrm{apoE}^{+/+}$counterparts (Figure $1 \mathrm{~A})$. The distribution of the subsets was not altered in apoE $\mathrm{E}^{-/-}$ mice maintained on a standard chow diet, but those fed a highfat diet showed a shift toward an increased frequency of Ly-6 $\mathrm{Chi}^{\text {hi }}$ monocytes $\left(\mathrm{CCR} 2^{+}\right)$compared with Ly-6Clo monocytes (CCR2-, CX3CR1 ${ }^{\text {hi) }}$ (Figure 1A). When C57BL/6 apoE ${ }^{+/+}$WT mice were fed the same fat-enriched diet, no alterations in monocyte numbers or subset distribution were observed (data not shown), suggesting that these changes in monocyte subsets were disease driven.

CD11c is a marker for mouse DCs (22). As shown previously (18), Ly-6Clo monocytes, but not Ly-6Chi blood monocytes, expressed CD11c. This pattern of CD11c in monocytes was not altered in apoE ${ }^{-/-}$mice (Figure $\left.1 \mathrm{~B}\right)$.

Labeling of blood monocyte subsets in apoE $E^{-/-}$mice. Tracing the fate of monocytes in mice is challenging, as no tracking method is without drawbacks. Following adoptive transfer, only a very small fraction of transferred monocytes can be recovered (16). Thus, large numbers of monocytes, pooled from many donors, are often transferred to overcome the relatively low sensitivity and quantitative nature of the method. Additional concerns include how isolation and manipulation of monocytes ex vivo affects subsequent differ- entiation and whether detection of a very small fraction of transferred cells accurately reflects behavior of the whole population (25). Nonetheless, the approach has demonstrated utility, including in studies of monocyte migration to atherosclerotic plaques (26). Knock-in of reporter genes to the CX3CR1 locus in mice (27) has revealed monocyte subsets that endogenously express distinct GFP intensity in blood $(15,16)$. Monocytes can be tracked in these mice without adoptive transfer (15), as we have previously done (19); however, such tracking is limited to a short term after extravasation of monocytes, since subsequent differentiation can either reduce GFP expression to undetectable levels, such as in macrophages (27), or elevate it, as in at least some DC populations (28). Moreover, the knock-in mice inherently lack at least 1 allele of CX3CR1. As we set out to study the role of CX3CR1 in monocyte trafficking and to examine time points beyond 1-2 days, these problems precluded the use of this approach.

We have recently developed techniques to selectively label endogenous Ly-6C hi or Ly- $6 C^{\text {lo }}$ monocytes i.v. through introduction of inert particulates $(24,29)$. These methods have the advantage of improved sensitivity and quantification of monocyte tracking over adoptive transfer, while yielding results that are in agreement with adoptive transfer approaches (29). Particles that lack TLR ligands do not stimulate p38 MAPK activation or degradation of IKB in cultured macrophages (30), so the extent of activation by inert latex beads in blood monocytes may be only minimal, but this is unclear. Although blood monocytes are predominantly labeled in the version of the method that labels Ly- $6 C^{\text {hi }}$ monocytes, a few neutrophils bear particles (24), and other organs such as spleen and bone marrow do at least transiently harbor particulate-labeled cells that are not monocytes, including neutrophils and B cells (24) (which are minimally or are not recruited to atherosclerotic plaques). Furthermore, labeling of Ly- $6 \mathrm{C}^{\text {hi }}$ monocytes is conducted following prior depletion of monocytes via use of apoptosisinducing liposomes (24), and the full effects of the prior depletion are also not yet known. This drawback is similar to that in transgenically engineered mice where cell populations, such as DCs (31) or macrophages (32), are induced to undergo selective apoptosis through genetic manipulation. In the case of clodronate-loaded liposomes, apoptosis of the targeted cell population is restricted to the locale of liposome administration (33), generally in contrast to transgenic models that lead to apoptosis systemically. However, so 


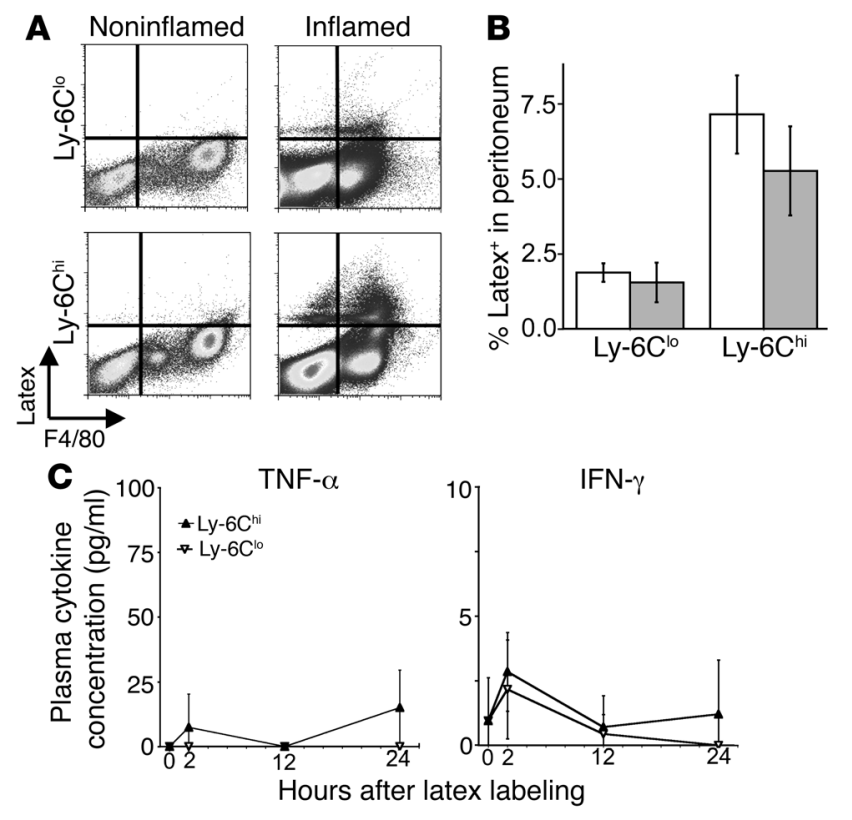

Figure 2

Recruitment of labeled WT monocytes to peripheral sites of acute inflammation. Ly- $6 \mathrm{C}^{\text {lo }}$ or Ly- $6 \mathrm{C}^{\text {hi }}$ monocytes in WT mice were labeled with latex (24). (A) The appearance of latex ${ }^{+}$cells in the peritoneal cavity was monitored by flow cytometry using F4/80 and CD115 antigens to identify monocytes and macrophages. Macrophages are seen as the prominent $\mathrm{F} 4 / 80^{\text {hi }}$ population in the noninflamed peritoneum (left dot plots). They become outnumbered by numerous infiltrating monocytes (inflamed plots; lower levels of F4/80 in lower right quadrants) and F4/80-CD115- neutrophils (inflamed plots; lower left quadrants). Note that Ly-6Chi labeling of monocytes leads to both latex+F4/80+ monocytes (inflamed plots; upper right quadrants) and latex ${ }^{+} \mathrm{F}^{4} / 80^{-}$ neutrophils (inflamed plots; upper left quadrants) appearing in the peritoneum, since some neutrophils transiently carry latex in this labeling protocol (24). In the inflamed peritoneum, latex ${ }^{+} L y-6 C^{\text {hi }}$ monocytes outnumbered latex ${ }^{+}$neutrophils $4: 1$, even though neutrophils dominate in acute peritoneal inflammation, in contrast to what occurs in atherosclerosis. (B) The graph shows the actual frequency of latex ${ }^{+} \mathrm{Ly}-6 \mathrm{C}^{10}$ or Ly-6C ${ }^{\text {hi }}$ monocytes in the inflamed peritoneum (gray bars), determined by flow cytometry, compared with the expected/known frequency (white bars) at which they would enter the peritoneum as unlabeled cells ("expected" data are derived from previously published calculations [ref. 16], as described in Methods). $n=4$ mice per group. Differences between the actual and expected frequencies were not significant. (C) Levels of plasma cytokines $( \pm S D)$ were measured at baseline or 2 , 12 , and 24 hours after administration of latex in the Ly- $6 C^{\text {hi }}$ or Ly- $6 C^{l o}$ monocyte labeling protocol. $n=3$ mice per time point.

far, no major problems with the effects of prior DC or macrophage apoptosis on interpretation of subsequent experiments in these approaches have been described.

Given these potential drawbacks, before using the particulate labeling method, we carried out experiments in WT mice to determine whether the labeling methods affected known trafficking patterns of Ly-6C $\mathrm{C}^{\text {hi }}$ and Ly-6C $\mathrm{C}^{\mathrm{lo}}$ monocytes or caused overt activation. We determined that engulfment of beads did not alter recruitment of monocytes to the acutely inflamed peritoneum, since accumulation of latex-labeled WT Ly-6C ${ }^{\text {lo }}$ or Ly- $6 C^{\text {hi }}$ monocytes in the thioglycollate-inflamed peritoneum (Figure 2A) mirrored the frequency predicted from studies that tracked unlabeled adoptively transferred Ly-6Chi and Ly-6Clo monocytes into the inflamed peritoneum (16) (Figure 2B). We were able to distinguish resident macrophages from newly entering monocytes, because the latter express lower levels of $\mathrm{F} 4 / 80$ than resident macrophages (34) (Figure 2A). This finding indicates that uptake of beads does not impair either subset of monocytes from emigrating out of the blood. In addition, migration of the latex ${ }^{+}$subsets was low to absent in the noninflamed peritoneum, where the major population was resident F4/80 hilatex ${ }^{-}$macrophages (Figure $2 \mathrm{~A}$ ), suggesting that the uptake of beads during labeling does not induce inflammatory patterns of extravasation.

To assess whether administration of latex beads i.v. leads to substantial activation of monocytes, we stained monocytes for activation markers. CD62L is readily shed upon cellular activation (35) and is expressed by Ly-6Chi but not Ly-6Clo monocytes (16). Staining for $\mathrm{CD} 62 \mathrm{~L}$ revealed that latex ${ }^{+} \mathrm{Ly}-6 \mathrm{C}^{\text {hi }}$ monocytes did not shed this activation-sensitive adhesion molecule (data not shown). Tissue factor is induced by many monocyte activation stimuli, but its expression and associated procoagulant activity were undetectable in the sera of latex-labeled mice (data not shown). Furthermore, we have carried out a whole mouse genome Affymetrix array (only 1 array has been completed in this ongoing analysis to date) by sorting latex ${ }^{-} \mathrm{Ly}-6 \mathrm{C}^{\text {hi }}$ and latex ${ }^{+} \mathrm{L} y-6 \mathrm{C}^{\text {hi }}$ monocytes from a pool of 5 female WT mice. The similarity of gene signatures, studied 24 hours after latex administration, between latex ${ }^{+}$and latex-Ly-6Chi monocytes isolated from the same mice was greater $\left(r^{2}=0.9984\right)$ than the natural biological variability observed in another gene array comparison of sorted Ly-6Chi monocytes from 2 separate sorts of unmanipulated mice $\left(r^{2}=0.9806\right)$. Thus, at least at the time point and condition examined to date, the carriage of latex bead(s) per se does not dramatically alter the monocyte.

We further reasoned that if overt activation is a major consequence of our labeling method, cytokine cascades associated with inflammation would be activated. Measuring cytokines in plasma would also allow us to detect whether any cells - not just monocytes - were activated enough to induce inflammatory gene expression. Thus, we measured the plasma levels of TNF- $\alpha$, IL- 6 , and IFN- $\gamma$ in naive animals (time 0 , baseline) or 2, 12, and 24 hours after latex was introduced i.v. IL- 6 was not detectable in any treatment (data not shown). Low levels of IFN- $\gamma$ were found, but these were similar to baseline levels (Figure 2C). Although levels of TNF- $\alpha$ were mildly increased in the Ly- $6 \mathrm{C}$ hi labeling protocol (Figure $2 \mathrm{C}$ ), the difference was not statistically significant compared with baseline, and levels were near the detection limit of the ELISA. Thus, the particle labeling protocols did not trigger strong signs of monocyte or systemic activation, suggesting that monocytes or any other cells were not grossly activated by encounter with the beads. Taking all these data together, we conclude that the 2 techniques of selectively labeling Ly- $6 \mathrm{C}^{\text {hi }}$ and Ly- $6 \mathrm{C}^{\text {lo }}$ monocytes, respectively, could permit the design of experiments to trace the fate of monocyte subsets in atherosclerotic lesions.

To study the entry of monocytes from the circulation into atherosclerotic lesions, we labeled blood monocyte subsets in apoE $\mathrm{E}^{-/-}$mice. Intravenous injection of $0.5-\mu \mathrm{m}$ latex particles efficiently and selectively labels monocytes in the circulation of WT mice (24). In WT mice, $10 \%-15 \%$ of the monocytes are phagocytically labeled using this approach, and even after 5-7 days following initial labeling, $5 \%-7 \%$ of blood monocytes remain latex ${ }^{+}(24)$. We applied this technique to apoE $\mathrm{E}^{-/-}$mice. After i.v. injection of latex, $1 \%-2 \%$ of blood leukocytes acquired the fluorescent beads within 1 day, and $90 \%$ of these latex ${ }^{+}$cells were CD $115^{+}$monocytes (Figure $3 \mathrm{~A}$ ). At day 1, 10\% 
A All cells Monocytes

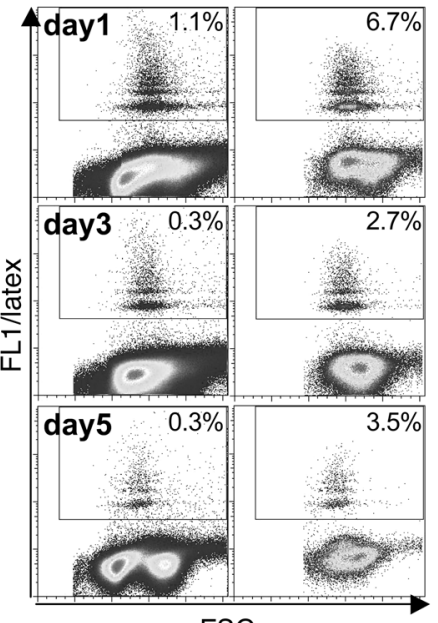

FSC
Latex ${ }^{+}$cells

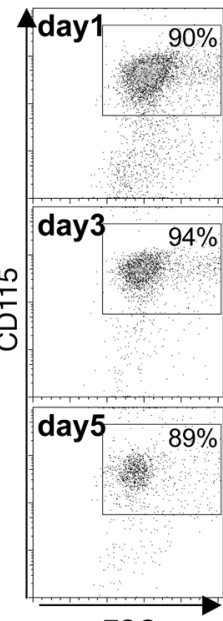

FSC
B

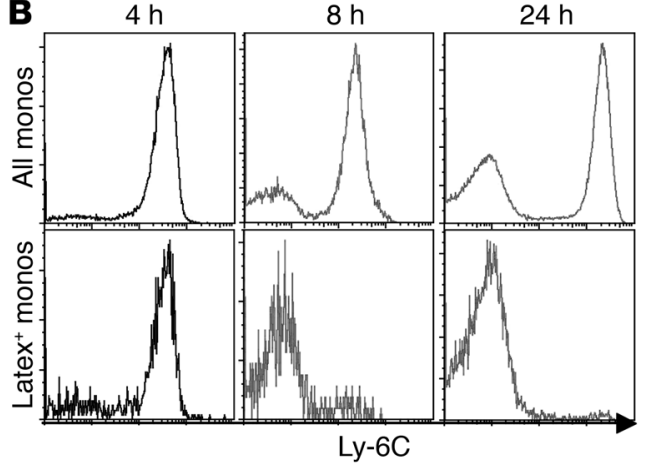

C $_{\text {Ly- } 6 C^{\prime}}$

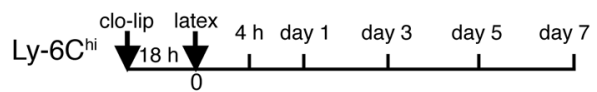

D $\quad 4 \mathrm{~h}$

Day 1

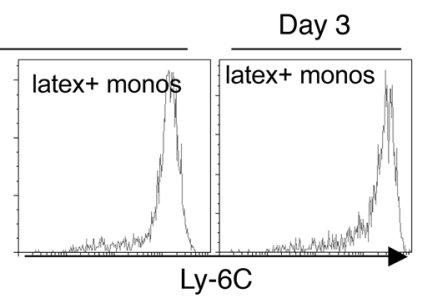

Ly-6C

Day 7

FSC

Day 5
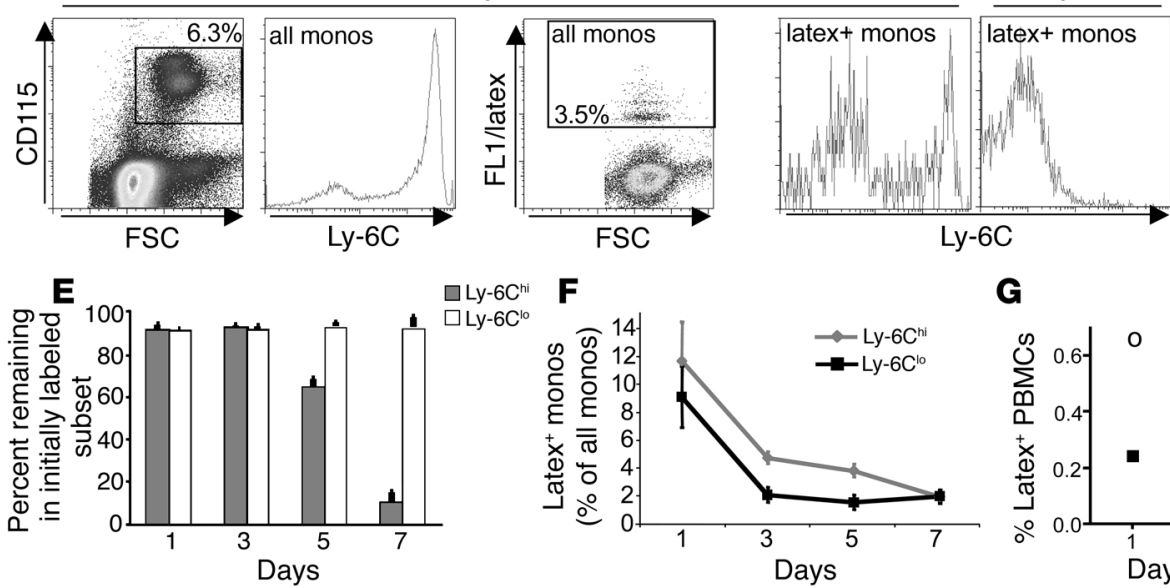

$F$

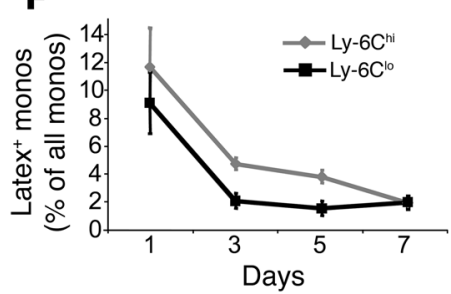

G

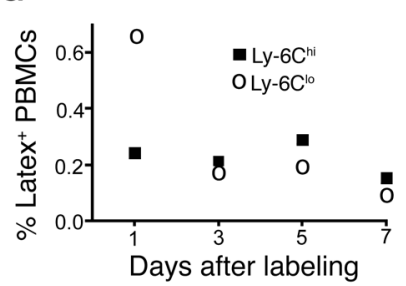

Figure 3

Labeling of monocyte subsets in apoE $E^{-/-}$mice. (A) Blood analysis after i.v. injection of latex beads in apoE $\mathrm{E}^{-/-}$mice. Representative plots $(\geq 5$ mice per time-point) at days 1-5 gated on all cells, monocytes, or latex cells. (B) Blood apoE ${ }^{-/-}$monocyte subsets 4,8 , or 24 hours after injection of latex: gating on all monocytes (All monos) or only latex ${ }^{+}$monocytes (Latex ${ }^{+}$monos); $n \geq 4$ mice per time point. (C) Schematic of injection protocols for labeling monocyte subsets. Injection of latex labeled Ly-6Clo monocytes; injection of clodronate-loaded liposomes (clo-lip) followed by latex 18 hours later labeled Ly-6Chi monocytes. (D) Blood analyses trace the depletion, reappearance, and subset of apoE ${ }^{-/-}$monocytes over time after i.v. injection of clo-lip followed by latex ( $n \geq 5$ per time point). (E) Latex ${ }^{+}$apoE ${ }^{-/-}$monocytes (\%) that remained Ly- $6 \mathrm{C}^{\text {hi }}$ or Ly-6Clo after clo-lip/latex or latex only injection, respectively. Reductions in frequency of Ly- $6 \mathrm{C}^{\mathrm{hi}}$ monocytes among circulating latex ${ }^{+}$cells reflected the extent of their conversion to Ly-6Clo monocytes. ( $\mathbf{F}$ and $\mathbf{G}$ ) Summary of the labeling efficiency using the 2 labeling techniques in apoE ${ }^{-/-}$mice (shown for 7-month-old mice on chow diet), presented as $(\mathbf{F})$ percentage of latex cells among all blood monocytes or $(\mathbf{G})$ normalized to account for the absolute frequency of apoE $\mathrm{E}^{-/}$monocytes after injection of latex i.v. (Ly-6Clo monocyte labeling) or latex i.v. following monocyte depletion (Ly-6Chi monocyte labeling). Normalized data were calculated by multiplying total frequency of the traced monocyte subset in the PBMC population by frequency of latex labeling within that subset.

(range, 6\%-13\%) of blood monocytes were latex ${ }^{+}$. The percentage of latex ${ }^{+}$monocytes decreased over time, more rapidly than in WT mice (24), to about $2 \%-2.5 \%$ (range $1 \%-3.5 \%$ ) of blood monocytes being latex ${ }^{+}$at days 3-7 (Figure 3A, and data not shown).
In apoE $\mathrm{E}^{-/-}$mice, like WT mice (24), i.v. injection of latex initially appeared in both monocyte subsets, but by 8 hours and thereafter, the vast majority of all circulating latex ${ }^{+}$monocytes were Ly- $6 \mathrm{C}^{\text {lo }}$ (Figure 3B) (24). During the first several hours that followed i.v. 


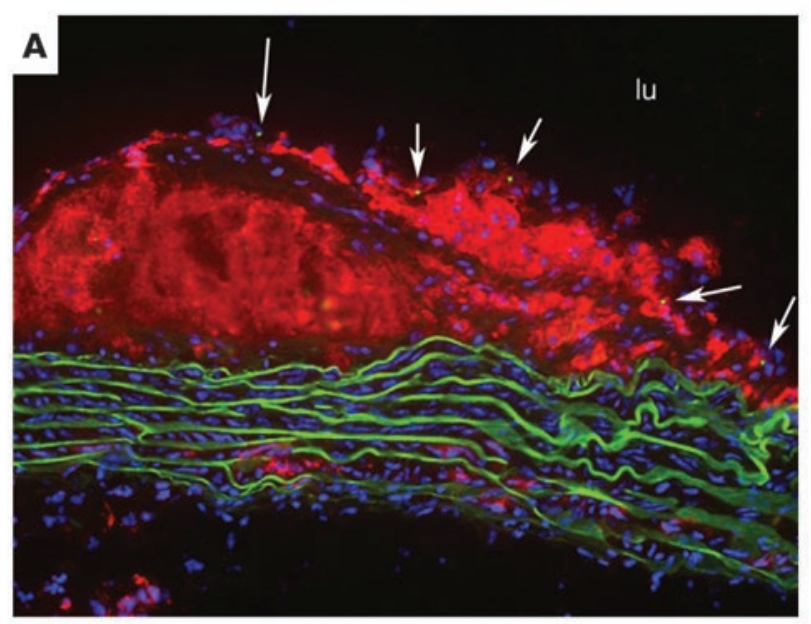

\section{B}
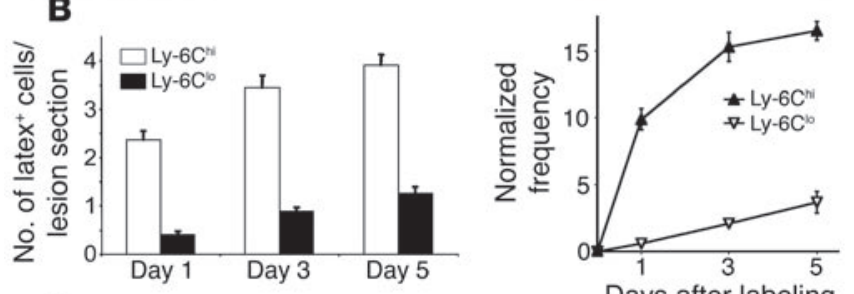

Days after labeling
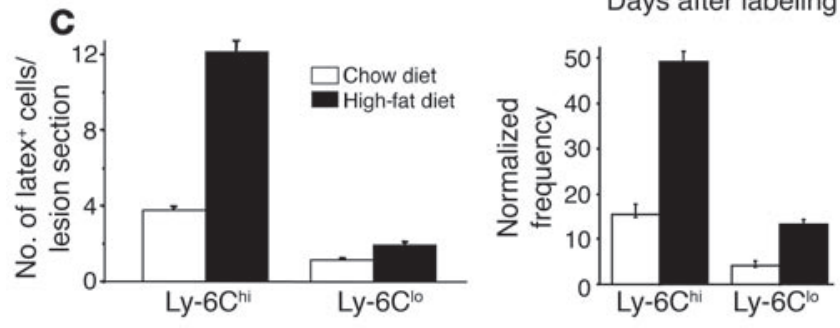

administration of latex beads, we noticed that Ly-6C lo monocytes in particular were often (Figure 3B), though not always (see Figure $1 \mathrm{D}$ in ref. 24), transiently reduced. It is likely that these monocytes marginated in the vasculature, suggesting that they were at least transiently activated by the injection of beads. Thus, i.v. injection of latex particles labels a portion of Ly- $6 \mathrm{C}^{\mathrm{lo}}$ monocytes (approximately $10 \%$ of total monocytes, or $20 \%$ of the Ly- $6 \mathrm{C}^{\text {lo }}$ subset) in apoE $\mathrm{F}^{-/}$ mice efficiently and specifically for more than 5 days (Figure 3C).

Ly-6Chi monocytes, on the other hand, can be stably labeled using a modification of this technique (Figure 3C), as previously described (24), wherein monocytes are transiently depleted using clodronate-loaded liposomes prior to introduction of latex beads. The i.v. injection of clodronate-loaded liposomes initially fully depleted blood monocytes in apoE $/ /$ mice (Figure 3D; 4 hours after i.v. injection of latex beads i.v., 22 hours after i.v. injection of clodronate-loaded liposomes). Neutrophils and B cells that carry latex in the absence of monocytes donate the latex to bone marrow monocytes (24), with a few neutrophils remaining latex ${ }^{+}$in the circulation (ref. 24 and Figure 2). Monocytes that returned to the circulation on day 2 were Ly-6Chi and 13\% (range, $8 \%-17 \%$ ) were latex ${ }^{+}$(Figure 3D). Latex ${ }^{+}$monocytes remained exclusively Ly- $6 \mathrm{C}^{\text {hi }}$ for 4 days. At day 5 , the percentage of latex ${ }^{+}$monocytes had decreased by more than half, and apparent conversion of these monocytes to the Ly-6C lo subset began to occur (18) and was completed by day 7 (Figure 3, E and F). Early in the Ly-6Chi

\section{Figure 4}

Tracing recruitment of monocyte subsets in atherosclerotic plaques. (A) Representative photomicrograph of a lesional section from a 7-month-old apoE ${ }^{-/-}$mouse where latex $+L y-6 C^{\text {hi }}$ monocyte-derived cells were observed. Red: CD68 ${ }^{+}$cells; blue: DAPI-stained nuclei; green: latex beads (internal elastic lamina is also green due to autofluorescence). Arrows indicate the presence of latex ${ }^{+}$cells within the lesion section. lu, lumen of aorta. Original magnification, $\times 260$. (B) Quantification of the recruitment of apoE $E^{-/-}$Ly-6Chi and Ly-6Clo monocyte subsets into lesions 1,3 , or 5 days after subset labeling in age-matched apoE $\mathrm{E}^{-/-}$mice (7 months) maintained on a chow diet. Mean number of latex ${ }^{+}$cells per lesion section is shown on the left, and normalized data that accounts for the differences in frequency of latex ${ }^{+}$monocyte subsets over time are shown on the right. (C) The effect of feeding a high-fat/high-cholesterol diet on the accumulation of apoE $\mathrm{E}^{-/-}$monocyte subsets into plaques 5 days after latex labeling. Mean number of latex ${ }^{+}$cells per lesion section is shown on the left, and normalized data that accounts for the differences in frequency of latex ${ }^{+}$monocyte subsets in response to diet are shown on the right. For all data points, 5-8 mice were studied.

labeling protocol, monocyte counts are reduced in the blood following depletion (24), such that the normalized frequency of latex ${ }^{+}$monocytes of each subset in the circulation indicates that latex ${ }^{+}$Ly- $6 C^{\text {lo }}$ monocytes were 3 -fold more frequent in blood than Ly-6Chi monocytes on the first day after labeling (Figure $3 \mathrm{G}$ ) following the respective labeling strategies. Data were normalized by evaluating the fraction of total PBMCs that was latex ${ }^{+}$at each time point, since total PBMC counts did not vary significantly in response to any of the experimental manipulations. By day 3 and thereafter, the normalized frequency of labeled monocytes was similar in the 2 subsets (Figure $3 \mathrm{G}$ ).

Both Ly-6C $C^{\text {bi }}$ and Ly-6Clo monocytes enter atherosclerotic plaques, but they differ in entry efficiency. We thus examined the capacity of the different monocyte subsets to enter and accumulate in atherosclerotic plaques. In mice in which monocyte subsets were labeled, we counted the number of latex ${ }^{+} \mathrm{CD} 68^{+}$cells in lesion sections after immunohistochemical staining of explanted aortic arches (Figure $4 \mathrm{~A}$ ) as a function of time (Figure 4B). Although lesion area differed between sections, the mean lesion area was similar among the different animals evaluated, since the mice were age and sex matched. Therefore, data were plotted as the number of latex ${ }^{+}$cells per lesion section. Given that only about $10 \%$ of monocytes were labeled with each technique (Figure 2), the actual number of newly entering monocytes in a given plaque would be proportionally higher. Both monocyte subsets entered the advanced atherosclerotic plaques of these mice. The initial entry of Ly- $6 \mathrm{C}^{\text {hi }}$ monocytes into lesions was approximately 20 -fold greater than the recruitment of Ly- $6 \mathrm{C}^{\text {lo }}$ monocytes, when normalized for the variances in frequency of labeled cells in blood using the 2 labeling protocols (Figure 4B). Ongoing accumulation of latex ${ }^{+}$monocytes was observed through the 5-day experimental time course. This accumulation increased linearly for Ly- $6 \mathrm{C}^{\text {lo }}$ monocytes but tapered in Ly- $6 \mathrm{C}^{\text {hi }}$ monocytes even after normalization.

The rates at which both Ly- $6 \mathrm{C}^{\text {hi }}$ or Ly- $6 \mathrm{C}^{\text {lo }}$ monocytes were recruited and retained in plaques was increased by 3 -fold (after normalization) in mice fed a fat-rich diet (Figure 4C), consistent with another recent study (26).

Distinct plaque phenotypes of recruited monocyte subpopulations. CD $11 \mathrm{c}^{+}$cells within plaques are poorly characterized, although they are known to be found in large numbers in many areas of 

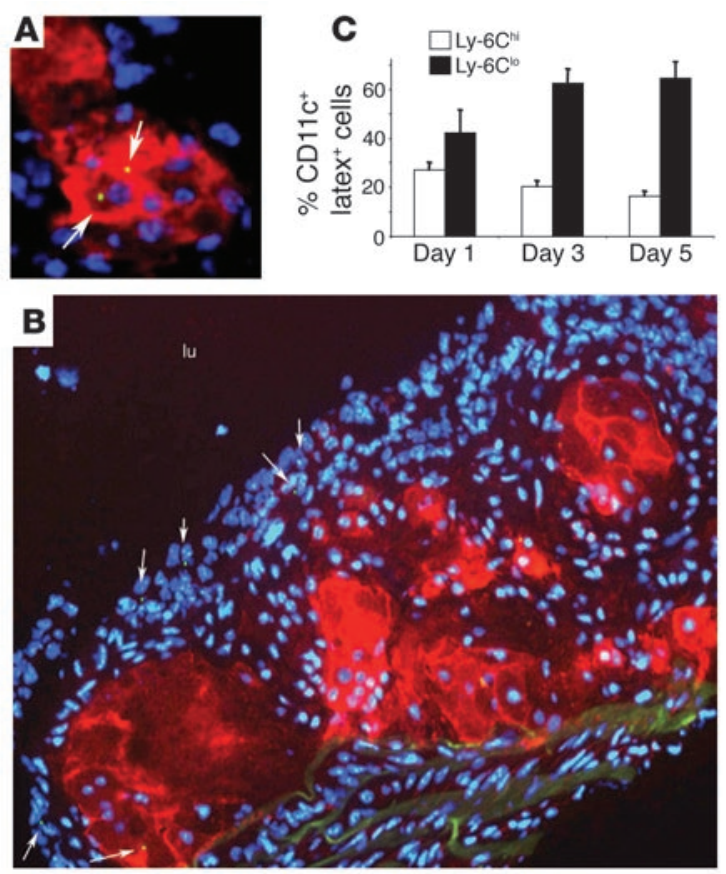

\section{Figure 5}

Phenotype of invading monocytes within atherosclerotic lesions of apoE $\mathrm{E}^{-/-}$mice. (A and $\mathbf{B}$ ) CD11 $\mathrm{C}^{+}$cells (red) in lesions (A, from Ly-6Clo labeling; original magnification, $\times 260$; $\mathbf{B}$, from Ly- $6 C^{\text {hi labeling; original }}$ magnification, $\times 130$ ). In areas where plaques were more advanced, CD $11 \mathrm{c}^{+}$cells typically surrounded and coalesced with necrotic cores that lacked intact nuclei (identified by DAPI staining in blue). Some CD $11 \mathrm{C}^{+}$cells were multinucleated. Cells bearing latex beads (green) are indicated by arrows. (C) The fraction of each latex ${ }^{+}$monocyte subset that colocalized with CD11c in lesion sections after monocyte labeling. For each time point and condition, 3 or more mice were individually analyzed, and approximately 50 sections per animal were scored.

plaque $(20,21)$. Immunostaining for these cells revealed that in some plaques areas, almost all CD $68^{+}$cells costained with CD11c (20). However, in other areas, only some $\mathrm{CD}^{+} 8^{+}$cells were $\mathrm{CD} 11 \mathrm{c}^{+}$. In these areas, $\mathrm{CD} 11 \mathrm{c}^{+}$cells were often found in clusters (Figure 5, $\mathrm{A}$ and $\mathrm{B}$ ). Some were very large cells that appeared multinucleated and/or closely associated with developing necrotic cores (Figure $5 B$ ). When Ly-6Chi monocytes were labeled with latex, $20 \%-30 \%$ of latex ${ }^{+}$cells in atherosclerotic plaques coexpressed CD11c, whereas approximately $60 \%$ of latex ${ }^{+}$cells in atherosclerotic plaques were CD11 $\mathrm{c}^{+}$when the latex labeling traced Ly-6C ${ }^{\text {lo }}$ monocytes (Figure $5 \mathrm{C}$ ), suggesting that the latter subset is more predisposed than classical CCR2 ${ }^{+}$monocytes to become CD $11 \mathrm{c}^{+}$cells in lesions.

Ly-6C hi , but not Ly-6C lo, monocytes employ CCR2 and CX3CR1 to enter atherosclerotic plaques. We next studied the chemokine receptors used by monocyte subsets to enter and accumulate within atherosclerotic plaques. We expected Ly-6 $\mathrm{C}^{\mathrm{hi}}$ monocytes to use CCR2 to enter lesions. It is thought that Ly-6 $\mathrm{C}^{\text {lo }}$ monocytes, which lack CCR2 $(15,16)$, utilize CX3CR1 for migration (16), but Ly-6C hi monocytes also express substantial levels of CX3CR1 (16). Because both CCR2-deficient and CX3CR1-deficient mice have reduced plaque development when crossed with apoE-deficient mice $(2,10$, 11 ), we could not readily directly compare the rate of monocyte influx in these strains. Instead, we designed an experimental plan that permitted us to study the recruitment of WT, CCR2-deficient, and CX3CR1-deficient monocytes into atherosclerotic plaques that were derived uniformly from apoE-deficient CCR2 ${ }^{+}$CX3CR $1^{+}$ mice. Specifically, we surgically transferred donor atherosclerotic aortic arches (36) from apoE $\mathrm{E}^{-/}$mice $\left(\mathrm{CCR} 2^{+} \mathrm{CX} 3 \mathrm{CR} 1^{+}\right)$into recipient mice that were $\mathrm{CCR} 2^{+/+} \mathrm{CX} 3 \mathrm{CR} 1^{+/+}$(WT), CCR2 $2^{-/-} \mathrm{CX} 3 \mathrm{CR} 1^{+/+}$, or $\mathrm{CCR} 2^{+/+} \mathrm{CX} 3 \mathrm{CR} 1^{-/}$and whose monocytes had been labeled with latex. Ly-6Chi monocyte subset labeling efficiency did not differ in WT and KO mice (data not shown). Labeling of Ly-6Clo monocytes was similar in CX3CR $1^{-/-}$Ly-6C lo and WT mice, but Ly-6C ${ }^{\text {lo }}$ monocytes showed reduced labeling in CX3CR1 $1^{-/}$mice when carboxylate-modified beads were used, although this difference was not significant when plain polystyrene beads were used (as in the lesion analysis). However, CCR2 $2^{-/-}$mice had fewer circulating Ly-6Chi monocytes, as reported previously $(19,37)$.

As expected, latex ${ }^{+}$CCR2 $2^{-/-}$Ly-6C hi monocytes were markedly less efficient than WT latex ${ }^{+} \mathrm{Ly}-6 \mathrm{C}^{\text {hi }}$ monocytes at entering and accumulating within the grafted plaques (Figure 6A). The mean reduction in latex ${ }^{+} \mathrm{CCR} 2^{-/-}$Ly-6 $\mathrm{C}^{\text {hi }}$ monocytes in plaques was $72 \%$, compared with WT controls (Figure 6A, left panel). However, when we accounted through normalization for the fact that Ly-6Chi monocytes exit the bone marrow less efficiently than WT monocytes, resulting in reduced numbers of circulating latex ${ }^{+}$ monocytes, there was only a $44 \%$ decrease in monocyte entry into plaques (Figure 6A, right panel), suggesting that CCR2 has 2 roles in mediating monocyte accumulation in atherosclerotic plaques: one effect is at the level of monocyte exit from the bone marrow; the other is emigration from blood into plaques.

Latex $^{+}$CCR2-/- Ly-6Clo monocytes entered plaques as well as WT monocytes (Figure 6B), which was expected, since this subset does not express CCR2 (16). Unexpectedly, however, latex ${ }^{+} L y-6 C^{\text {lo }}$ monocytes also did not require CX3CR1 to emigrate into plaques (Figure 6B), but latex ${ }^{+} \mathrm{Ly}-6 \mathrm{C}^{\text {hi }}$ monocyte entry into plaques was reduced by $54 \%$ in the absence of CX3CR $1(P<0.02)$ (Figure $6 \mathrm{~A}$ ). Thus, Ly-6Chi blood monocytes utilize both CCR 2 and CX3CR1 to enter atherosclerotic lesions. The CX3CR1 ligand CX3CL1 was detected on the endothelium overlying plaques (Figure 6C), consistent with the possibility that monocyte CX3CR1 may bind CX3CL1 on endothelium.

Role of CCR5 in monocyte migration to plaques. To identify chemokine receptors that $\mathrm{Ly}-6 \mathrm{C}^{\mathrm{lo}}$ monocytes use to enter plaques, we examined data from an ongoing preliminary gene array analysis that compared gene expression among monocyte subsets. CCR5 was selectively upregulated in apoE ${ }^{-/-}$Ly-6Clo monocytes (data not shown), consistent with reports that the human $\mathrm{CD}^{+} 6^{+}$counterparts to these mouse monocytes preferentially express elevated levels of CCR5 (38). To verify selective CCR5 upregulation in apoE-deficient Ly-6Clo monocytes, we conducted real-time PCR for CCR5 and other chemokine receptors from sorted monocyte subpopulations. Indeed, CCR5 mRNA was selectively induced in Ly-6Clo monocytes from apoE-/- mice (Table 1). Cell-surface expression of CCR5 was very weak relative to control staining in $\mathrm{Ly}-6 \mathrm{C}^{\mathrm{hi}}$ monocytes, but expression was higher in Ly-6Clo monocytes (Figure 7A). To determine whether CCR5 participated importantly in mediating entry or accumulation of monocyte subsets into plaques, we treated cohorts of apoE-/- mice with anti-CCR5 neutralizing $\mathrm{mAb}(39)$ or isotype control. Anti-CCR5 did not deplete circulating monocytes and did not significantly alter the frequency of Ly- $6 C^{\text {hi }}$ or Ly-6Clo blood monocytes containing or lacking latex beads (data not shown). Nonetheless, there was a statistically significant inhibition of approximately $50 \%$ in Ly-6Clo monocyte 


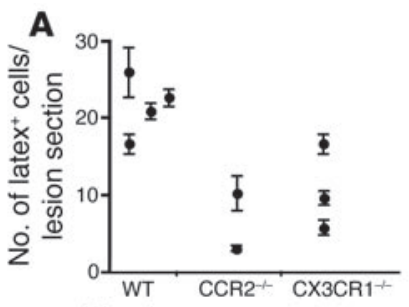

Blood monocyte genotype

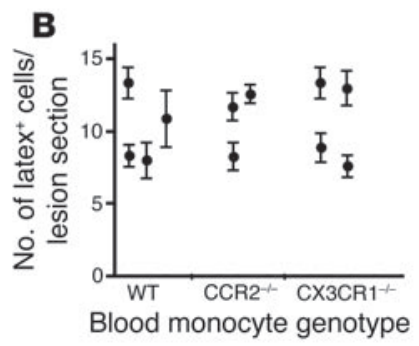

C
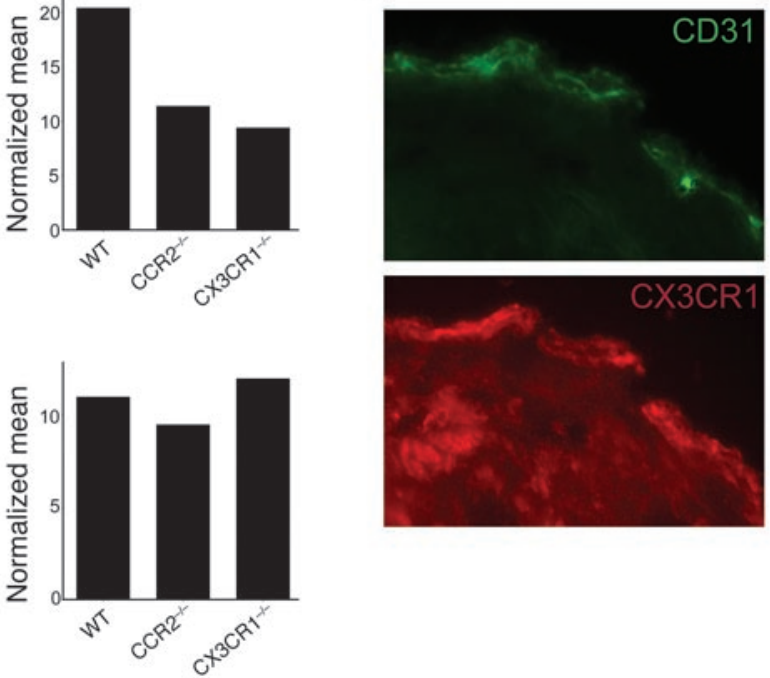

Figure 6

Chemokine receptor utilization for entry of monocytes into atherosclerotic lesions. Atherosclerotic aortic arches from apoE ${ }^{-/-}$mice were surgically transplanted into WT, CCR2 $2^{-1}$, or CX3CR $1^{-1-}$ mice 1 day after $L y-6 C^{\text {hi }}$ or $L y-6 C^{l o}$ blood monocytes had been labeled, respectively. Recruitment of latex ${ }^{+}$ monocytes into the aortic graft was analyzed 3 days later. (A) Ly- $6 C^{h i}$ and (B) Ly-6Clo monocyte trafficking. Plots show the number of latex ${ }^{+}$cells in atherosclerotic lesion sections (left; mean \pm SEM shown for each animal studied) or the normalized mean values that take into account frequency of circulating latex ${ }^{+}$monocytes (right; values from all animals are averaged) after Ly- $6 \mathrm{C}^{\text {hi }}$ or Ly- $6 \mathrm{Cl}^{l o}$ monocyte labeling in mice that received transplants of apoE-/- aortic arches containing atherosclerotic lesions. (C) Immunostaining of lesion section to identify CD31+ endothelial cells (green) and CX3CL1 (red). Original magnification, $\times 130$.

entry into plaques (Figure 7B). Thus, although Ly-6C lo monocytes do not use CCR2 or CX3CR1 to enter atherosclerotic plaques, they are partially dependent upon CCR5 to do so. However, the antibody also partially blocked Ly-6Chi monocyte entry into or early accumulation within plaques (Figure 7C), even though it is less obvious that Ly-6Chi monocytes expressed CCR5. Thus, the effect of the anti-CCR5 neutralizing $\mathrm{mAb}$ was not limited to regulation of the trafficking of Ly-6Clo monocytes.

\section{Discussion}

Here we applied techniques for differentially labeling mouse monocyte subsets with latex beads administered i.v. in order to uncover details regarding monocyte recruitment to atherosclerotic plaques. The experiments we describe herein suggest that carriage of latex beads as a tracer has minimal effects on monocyte traf- ficking and activation, but it is important to point out that the effects on monocytes, or other systemic effects, of our methods are not fully known, as to date we have only a partial understanding of the mechanisms of labeling (24). Our approach reveals that both major subsets of monocytes enter atherosclerotic plaques, with a greater rate of recruitment of the "inflammatory" Ly-6Chi CCR2 ${ }^{+}$monocyte subset (also called classical monocytes).

CCR2 and CX3CR1 are the major chemokine receptors used to classify monocyte subsets $(16,17)$. Here we assessed the role of these chemokine receptors in mediating the recruitment of the different subsets of monocytes into plaques. It is perhaps not surprising that we observed a strong dependency of classical monocytes on CCR2 for entry into plaques. However, it was unexpected that this same subset of monocytes would depend also on CX3CR1 to emigrate into lesions. By contrast, the prediction that $\mathrm{Ly}-6 \mathrm{C}^{\mathrm{lo}} \mathrm{CCR} 2^{-}$monocytes (termed nonclassical) would instead employ CX3CR1 to enter plaques, based on previous literature suggesting that nonclassical monocytes use CX3CR1 for trafficking (16), was not correct. These nonclassical monocytes entered plaques in a CCR2and CX3CR1-independent manner and instead used CCR5 for trafficking into plaques. The role of CCR5 was not specific to Ly-6Clo monocytes, as neutralizing this chemokine also partially blocked Ly-6Chi monocytes, further emphasizing the point that multiple chemokine receptors operate in concert to control the migration of a single population of cells. Future studies will be needed to determine whether anti-CCR5 acts directly on monocytes or affects monocytes indirectly through modulation of other $\mathrm{CCR}^{+}$cells.

Mice lacking CX3CR1 and CX3CL1 were described previously and had no notably distinct phenotype compared with WT counterparts (40). In acute inflammatory assays, such as inflammation induced by thioglycollate injection into the peritoneum, CX3CR1 was not required (27), in contrast to CCR2 (41-43). Moreover, CX3CR1 was not required for responses to contact sensitizers,

\section{Table 1}

Results of real-time PCR comparing chemokine receptor mRNA among monocyte subsets

\begin{tabular}{|c|c|c|c|c|c|c|}
\hline & WT Ly-6C'10 & WT Ly-6Chi & Chow ${ }^{A}$ Ly-6Clo & Chow Ly-6Chi & $\operatorname{Diet}^{\mathrm{B}} \mathrm{Ly}-6 \mathrm{C}^{10}$ & Diet Ly-6Chi \\
\hline Cor1 & 8.9 & 28.5 & 5.5 & 23.7 & 5.3 & 73.5 \\
\hline Ccr2 & 144.1 & 706.0 & 144.4 & $1,337.1$ & 122.5 & $1,396.2$ \\
\hline$C \times 3 c r 1$ & $1,342.1$ & 740.7 & $1,694.2$ & 772.4 & $1,633.0$ & 786.3 \\
\hline Ccr5 & 12.4 & 10.4 & 35.7 & 11.6 & 32.8 & 12.9 \\
\hline
\end{tabular}

The relative expression of each CCR was calculated against the relative abundance of cDNA encoding for ubiquitin. Using this method, we were able to discern relative differences in copy number for a given cDNA, but comparisons between different cDNAs (i.e., relative expression of CCR2 to CCR5) were not valid. AChow refers to 6-month-old apoE ${ }^{-/}$mice maintained on a standard chow diet. BDiet refers to 6-month-old apoE--/- mice maintained on a high-fat diet from 6 weeks of age. 
A
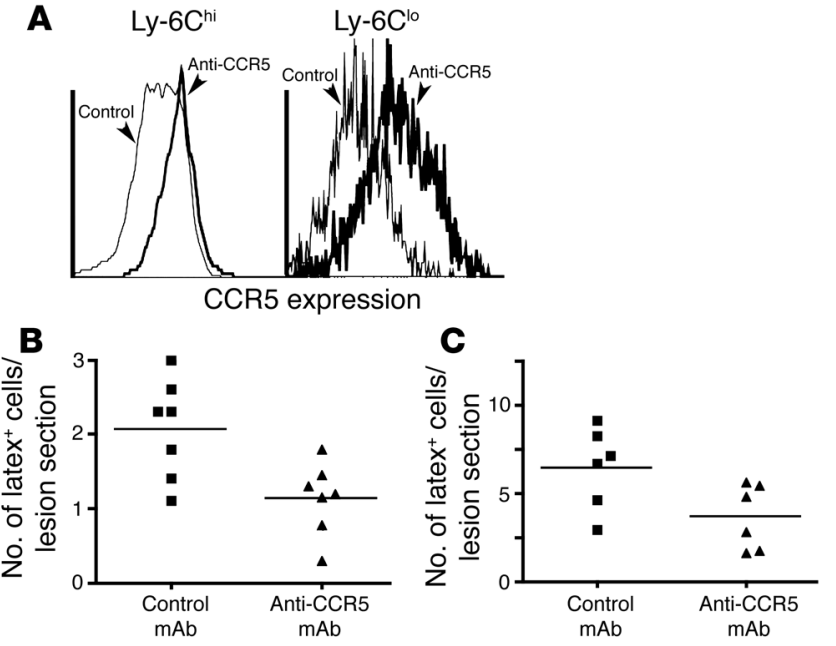

Figure 7

Role of CCR5 in monocyte migration into plaques. (A) Blood monocyte subsets were stained for CCR5 expression in apoE $\mathrm{E}^{-/-}$mice. Bold line, staining with anti-CCR5 $\mathrm{mAb}$; thin line, isotype control staining. (B) apo $\mathrm{E}^{-/-}$mice were treated i.v. with latex to label $\mathrm{Ly}-6 \mathrm{C}^{10}$ monocytes. The mean number of latex ${ }^{+}$cells in lesion sections collected along the entire aortic arch of apoE $\mathrm{E}^{-/-}$mice treated with control mAb or anti-CCR5 was determined by an observer blinded to the experimental protocol. Each data point represents analysis of an individual mouse. Differences between control and anti-CCR5 were significant; $P<0.01$. (C) An analysis similar to that represented in $\mathbf{B}$, except apoE $\mathrm{E}^{-/-}$mice were treated so that $\mathrm{Ly}-6 \mathrm{C}^{\mathrm{hi}}$ monocytes were latex ${ }^{+}$during the assay. Differences between control and anti-CCR5 were significant; $P<0.04$.

peripheral nerve injury, or challenge with Toxoplasma gondii (27). CCR2, by contrast, is needed for migration of monocytes in these and other reactions to injury $(2,44,45)$. Thus, CX3CR1 may be more selectively involved in recruitment of inflammatory monocytes into atherosclerotic plaques, but by contrast CCR2 is widely required by monocytes in nearly all models of inflammation. Therapeutics designed to block plaque growth by inhibiting monocyte recruitment would be expected to have fewer side effects if they targeted a molecular event more selective for plaque development, instead of a pathway that was universally required for monocyte recruitment to sites of injury. Thus, we propose that the use of antagonists of CX3CR1 may be more favorable for reducing the advance of atherosclerotic disease than antagonists of CCR2.

Indeed, our findings that classical monocytes unexpectedly utilize CX3CR1 to enter atherosclerotic plaques may explain in large part the role of CX3CR1 in the disease. CX3CR1 and CX3CL1 are known to be involved in atherosclerosis (10-12), but the steps at which these molecules acted and the cell types that required these molecules for plaque growth had not been identified. Although some models predicted that CX3CR1 was involved in monocyte recruitment (13), others favored a role for CX3CR1 in smooth muscle cell biology $(14,46)$. We cannot eliminate the possibility that CX3CR1 is needed by smooth muscle cells to promote cardiovascular disease, but we show for the first time to our knowledge a direct role for this molecule in monocyte recruitment.

That deficiencies in either CCR2 or CX3CR1 greatly reduce the entry of classical monocytes into atherosclerotic plaques indicates that these chemokine receptors have nonredundant roles and may act at different stages during recruitment. Recently, it has been revealed that CCR2 mediates monocyte egress from the bone marrow (37), a step far upstream of direct monocyte entry into plaques. However, since we were able to normalize for the decrease in circulating monocytes by quantifying latex ${ }^{+}$monocytes in the blood, our findings indicate that CCR2 is also important for migration of monocytes at a step downstream of exit from the bone marrow. Another recent study showing that signals through CCR2 can increase monocyte binding to CX3CL1 fits very well with our findings and offers potential mechanistic insight as to how these chemokine receptors can work together in the same cell type (47).

It is important to note that the extent of the block that we observed in monocyte recruitment to plaques when monocytes lacked CX3CR1 was greater than the overall reduction in lesion area in apoE $\mathrm{E}^{-/-} \times \mathrm{CX} \mathrm{CR} 1^{-/-}$mice or in $\mathrm{apoE}^{-/-} \times \mathrm{CX}^{-1} \mathrm{CL} 1^{-/-}$mice. Our assays were short term ( $\leq 5$ days), but disease develops and progresses over weeks, months, and years. Thus, we anticipate that if given more time, more monocytes would be able to overcome deficiency in CX3CR1 and enter plaques. From a therapeutic standpoint, total abrogation of monocyte recruitment may not be needed to achieve benefit, and the polymorphism in CX3CR1 in the human population that leads to relative protection from cardiovascular disease (8) supports this idea. However, human atherosclerotic plaques may have only low levels of or no CX3CL1 expressed in the arterial endothelium, as its expression is not readily detected on the arterial endothelium $(46,48)$. The failure to detect CX3CL1 in these studies may relate to the sensitivity of the assay for detection, but we cannot exclude the possibility that human lesions differ from those in mice and do not express CX3CL1 in the arterial endothelial compartment in vivo.

Besides providing an analysis of the role of chemokine receptors in monocyte recruitment to atherosclerotic plaques, our study examines aspects of macrophage heterogeneity within plaques. A phenotypic difference retained in plaques between recruited Ly-6Chi and Ly-6Clo monocytes is that the latter express CD $11 \mathrm{c}$ in lesions more frequently. This differential expression may be a marker for functional differences between the 2 populations within plaques. CD11c is widely expressed in atherosclerotic plaques of mice $(20,21)$ and colocalizes with CD68, a marker often used to delineate macrophages. However, there are also many $\mathrm{CD}^{+} 8^{+}$ cells in lesions that are $\mathrm{CD} 11 \mathrm{c}^{-}$, reflecting a more traditional macrophage phenotype. CD11c is typically regarded as a DC marker $(31,49)$, and in many tissues, it is restricted to DCs. The major exception to the association of CD11c with DCs is its expression by lung macrophages. We find that Ly- $6 \mathrm{C}^{\mathrm{lo}}$ monocytes are particularly predisposed to becoming $\mathrm{CD} 11 \mathrm{c}^{+}$cells within lesions. Thus, if these lesional cells are DCs, they are likely to arise not from a DC precursor independent of monocytes but instead from $\left(\mathrm{Ly}-6 \mathrm{C}^{\mathrm{lo}}\right.$ ) monocytes. Future studies will be focused on further characterizing this DC-like population in an effort to determine their role in disease compared with that of traditional CD11 $\mathrm{c}^{-}$macrophages.

\section{Methods}

Mice. Standard (Ly5.2; CD45.2) C57BL/6 mice, congenic (Ly5.1; CD45.1) C57BL/6 mice, and Ly5.2 apoE $\mathrm{E}^{-/-}$mice were purchased from Taconic or Jackson Laboratory. Congenic Ly5.1 (CD45.1) apoE-/- mice were bred and maintained in our colony. Some apoE $\mathrm{E}^{-/}$mice were transitioned at 5 weeks of age to a high-fat diet ( $21 \%$ milk fat, $0.15 \%$ cholesterol; Harlan Teklad). CCR2 $-/-$ mice (42) and CX3CR $1^{-/-}$mice (27) were also maintained in our colony. All mice were fully backcrossed at least 10 generations on the C57BL/6 background. They were housed in a pathogen-free environment 
at Mount Sinai School of Medicine and used in accordance with protocols approved by the Institutional Animal Care and Utilization Committee.

Labeling of blood monocytes and normalization of data. 0.5- $\mu \mathrm{m}$ Fluoresbrite FITC-dyed (YG) plain microspheres or $0.5-\mu \mathrm{m}$ Fluoresbrite polychromatic red microspheres (2.5\% solids [wt/vol]; Polysciences Inc.) were diluted 1:25 in $\mathrm{PBS}$, and $250 \mu \mathrm{l}$ of the solution was injected into the lateral tail vein of mice in order to selectively label Ly-6C $\mathrm{C}^{\text {lo }}$ monocytes (24). For selective labeling of Ly-6Chi monocytes (24), $250 \mu \mathrm{l}$ of liposomes containing clodronate (33) were i.v. injected in order to transiently deplete monocytes, followed by $250 \mu \mathrm{l}$ fluorescent microspheres i.v. 16-18 hours later. Clodronate was a gift from Roche and was incorporated into liposomes as described previously (33).

To normalize data wherein different monocyte subsets were labeled or different strains of mice were used, we calculated the frequency of total labeled monocytes of each subset in the circulation. To do this for each animal at each time point available, we multiplied the percentage of blood PBMCs that were $\mathrm{Ly}-6 \mathrm{C}^{\mathrm{hi}}$ or Ly- $6 \mathrm{C}^{\text {lo }}$ monocytes (depending on the subset being traced) by the percentage of latex ${ }^{+}$monocytes. This value represents the percentage of latex ${ }^{+}$PBMCs and is a reliable normalization factor because total PBMC counts did not vary significantly in response to any of the experimental manipulations. We then took a mean value of the relevant time points available. For example, for day- 5 analyses, we took the mean of normalized frequencies from days 1,3 , and 5 (see Figure 3 F). For day- 3 analyses, we took the mean of the normalized frequency from day 1 and 3 . We divided the number of latex ${ }^{+}$monocytes enumerated in plaque sections by this value to derive a normalized value quantifying recruitment into plaques.

Surgical transfer of the aortic arch. One day prior to surgical transfer of atherosclerotic aortic arches to recipient mice, monocyte subsets of the designated recipients were labeled with latex beads as described above. Atherosclerotic aortic arches from $\mathrm{CD} 45.1^{+}$apoE-/- mice, kept on a hypercholesterolemic/ high-fat diet for 12 weeks, were surgically transplanted into the abdominal aorta of WT, CCR2 $2^{--}$, or CX3CR1 1 - mice, as previously described $(20,36)$. In this protocol, lesions rapidly regress, but previous studies have shown that the rate of incoming (in contrast to exiting) monocytes is not altered in the first 3-day window after surgery (20). Two to 4 surgeries were performed on a given day, and the recipients on each day of surgery always included matched representatives of WT and either CCR2/-- or CX3CR1/- genotypes, allowing comparison between genotypes in side-by-side processing.

Effect of anti-CCR5 $m A b$ on monocyte recruitment to plaques. apoE ${ }^{-/-}$mice (7 months of age, maintained on a normal chow diet) were administered latex beads i.v. (24) simultaneously with $50 \mu \mathrm{g}$ i.p. of anti-CCR5 mAb MC-68 (39) or $50 \mu$ i.p. of rat IgG2b (catalog MAB0061; R\&D Systems). For mice wherein Ly-6Chi monocytes were to be latex labeled, clodronateloaded liposomes were administered 1 day prior to latex and $\mathrm{mAb}$ injection. All mice were given a second administration of mAb i.p. 2 days after the initial injection. Mice were sacrificed 5 days after the first administration of $\mathrm{mAb}$. Aortic arches were prepared and analyzed in a blinded manner as per the protocol described in "Immunostaining," below.

Model of acute inflammation in the peritoneal cavity. Inflammation was induced by i.p. injection of $1 \mathrm{ml} 4 \%$ thioglycollate (Sigma-Aldrich). After 18 hours, cells in the peritoneal lavage were stained for F4/80 and CD115. The actual frequency of latex ${ }^{+} \mathrm{Ly}-6 \mathrm{C}^{\text {lo }}$ or Ly- $6 \mathrm{C}^{\text {hi }}$ monocytes entering the inflamed peritoneum was compared with the expected frequency of entry if migration was normal. This expected frequency was determined by studying how efficiently each monocyte subset exited blood and entered the inflamed peritoneum after adoptive transfer, as previously reported (16). For example, the published data regarding the frequency of Ly- $6 \mathrm{C}^{\mathrm{lo}}$ monocyte entry in the thioglycollate-inflamed peritoneum were calculated as follows (16): (600 Ly-6C lo monocytes/2,200 total monocytes in peritoneum after adoptive transfer $) \div\left(1,600 \mathrm{Ly}-6 \mathrm{C}^{\text {lo }}\right.$ monocytes $/ 650$ Ly- $6 \mathrm{C}^{\text {hi }}$ monocytes in the circulation $)=0.11$, or $11 \%$. We thus multiplied $11 \%$ by the frequency of latex ${ }^{+}$blood Ly- $6 \mathrm{C}^{\text {lo }}$ monocytes for each animal in our study that were given thioglycollate to derive the predicted frequency of latex ${ }^{+} \mathrm{Ly}-6 \mathrm{C}^{\mathrm{lo}}$ monocytes entering the peritoneum, if carriage of beads did not impact trafficking. The actual frequency of peritoneal latex ${ }^{+} \mathrm{Ly}-6 \mathrm{C}^{\mathrm{lo}}$ monocytes was determined by examining the frequency of $\mathrm{F} 4 / 80^{+} \mathrm{CD} 115^{+}$latex ${ }^{+}$cells in the peritoneal lavage of Ly- $6 \mathrm{C}^{\text {lo }}$ latex-labeled mice using flow cytometry. A similar calculation was performed for Ly- $6 \mathrm{C}^{\text {hi }}$ monocytes.

Flow cytometry. Blood was drawn and prepared for staining as described previously (24). Staining for flow cytometric analysis was conducted using fluorochrome-conjugate combinations of the following mAbs: CD115, CD11c, CD45.1, and CD45.2 from eBioscience; F4/80 (AbD Serotec); Gr-1 (reacts with Ly-6C and Ly-6G, but only Ly-6C is expressed by monocytes), $\mathrm{CD} 11 \mathrm{~b}$, and CD62L from BD Biosciences - Pharmingen; or appropriate IgG isotype controls (BD Biosciences - Pharmingen or R\&D Systems). Detection of CCR5 expression involved staining with unconjugated MC-68 (39), followed by biotinylated anti-rat IgG2b (Jackson ImmunoResearch Laboratories Inc.) and streptavidin APC (CALTAG Laboratories).

Immunostaining. The aortic arch or the transplanted aortic graft was flushed with $25 \mathrm{ml}$ of PBS, excised, and frozen in OCT (Sakura). Sections of $8 \mu \mathrm{m}$ were collected (every fourth section kept) along the entire aortic arch and fixed with 3\% paraformaldehyde. All of the collected sections (typically about 50 per animal) were evaluated, including those lacking beads, and the mean number of latex ${ }^{+}$cells per section \pm SEM along the entire arch was determined. Immunofluorescence microscopy was performed using biotinconjugated CD11c (BD Biosciences - Pharmingen), biotinylated anti-mouse CX3CL1 polyclonal antibody (R\&D Systems), biotinylated normal goat IgG (R\&D Systems), or biotin-conjugated hamster IgG (BD Biosciences Pharmingen), followed by Streptavidin-Cy3 (Sigma-Aldrich). Identification of CD68 or CD31 was made using mAbs against these proteins from Serotec or rat IgG as the isotype control (R\&D Systems) followed by anti-rat Cy3 or Cy2 conjugates (Jackson ImmunoResearch Laboratories Inc.). The number of latex ${ }^{+}$cells per section was manually counted at high magnification.

PCR. For real-time PCR analysis of chemokine receptors expressed by sorted monocyte subsets, previously described methods were used (50).

ELISAs. ELISA kits to detect TNF- $\alpha$, IL-6, and IFN- $\gamma$ were purchased from R\&D Systems and used according to manufacturer's directions.

Statistics. All data are presented as mean \pm SEM or mean \pm SD. Two-tailed Student's $t$ test was used for comparisons between experimental groups. Significant differences were defined at $P<0.05$.

\section{Acknowledgments}

This work was supported by NIH grants AI49653 and HL69446 to G.J. Randolph and DK067381 to S.A. Lira and funds from the German Research Foundation to A.J. Habenicht. F. Tacke was supported by a postdoctoral fellowship from the German Research Foundation, C. Jakubzick by a training grant from the NIH, and D. Alvarez by a postdoctoral fellowship from Canadian Institutes of Health Research.

Received for publication March 16, 2006, and accepted in revised form October 24, 2006.

Address correspondence to: Gwendalyn J. Randolph, Department of Gene and Cell Medicine, Mount Sinai School of Medicine, 1425 Madison Ave., Box 1496, New York, New York 10029, USA. Phone: (212) 659-8262; Fax: (212) 803-6740; E-mail: Gwendalyn. Randolph@mssm.edu.

Frank Tacke's present address is: Medical Clinic III, RWTH-University Hospital Aachen, Aachen, Germany. 
1. Gu, L., et al. 1998. Absence of monocyte chemoattractant protein-1 reduces atherosclerosis in low density lipoprotein receptor-deficient mice. $\mathrm{Mol}$. Cell. 2:275-281.

2. Boring, L., Gosling, J., Cleary, M., and Charo, I.F. 1998. Decreased lesion formation in CCR2-/- mice reveals a role for chemokines in the initiation of atherosclerosis. Nature. 394:894-897.

3. Huo, Y., et al. 2001. The chemokine KC, but not monocyte chemoattractant protein-1, triggers monocyte arrest on early atherosclerotic endothelium. J. Clin. Invest. 108:1307-1314. doi:10.1172/ JCI200112877.

4. Kuziel, W.A., et al. 2003. CCR5 deficiency is not protective in the early stages of atherogenesis in apoE knockout mice. Atherosclerosis. 167:25-32.

5. Zernecke, A., et al. 2006. Deficiency in CCR5 but not CCR1 protects against neointima formation in atherosclerosis-prone mice: involvement of IL-10. Blood. 107:4240-4243.

6. Potteaux, S., et al. 2005. Chemokine receptor CCR1 disruption in bone marrow cells enhances atherosclerotic lesion development and inflammation in mice. Mol. Med. 11:16-20.

7. Potteaux, S., et al. 2006. Role of bone marrowderived CC-chemokine receptor 5 in the development of atherosclerosis of low-density lipoprotein receptor knockout mice. Arterioscler. Thromb. Vasc. Biol. 26:1858-1863.

8. McDermott, D.H., et al. 2003. Chemokine receptor mutant CX3CR1-M280 has impaired adhesive function and correlates with protection from cardiovascular disease in humans. J. Clin. Invest. 111:1241-1250. doi:10.1172/JCI200316790.

9. Apostolakis, S., et al. 2006. Effects of polymorphisms in chemokine ligands and receptors on susceptibility to coronary artery disease. Thromb. Res. 119:63-71. doi:10.1016/j.thromres.2005.12.016.

10. Lesnik, P., Haskell, C.A., and Charo, I.F. 2003. Decreased atherosclerosis in CX3CR1-/- mice reveals a role for fractalkine in atherogenesis. J. Clin. Invest. 111:333-340. doi:10.1172/JCI200315555.

11. Combadiere, C., et al. 2003. Decreased atherosclerotic lesion formation in CX3CR1/apolipoprotein $\mathrm{E}$ double knockout mice. Circulation. 107:1009-1016.

12. Teupser, D., et al. 2004. Major reduction of atherosclerosis in fractalkine (CX3CL1)-deficient mice is at the brachiocephalic artery, not the aortic root. Proc. Natl. Acad. Sci. U. S. A. 101:17795-17800

13. Cybulsky, M.I., and Hegele, R.A. 2003. The fractalkine receptor CX3CR1 is a key mediator of atherogenesis. J. Clin. Invest. 111:1118-1120. doi:10.1172/ JCI200318237.

14. Bursill, C.A., Channon, K.M., and Greaves, D.R. 2004. The role of chemokines in atherosclerosis: recent evidence from experimental models and population genetics. Curr. Opin. Lipidol. 15:145-149.

15. Palframan, R.T., et al. 2001. Inflammatory chemokine transport and presentation in HEV: a remote control mechanism for monocyte recruitment to lymph nodes in inflamed tissues. J. Exp. Med. 194:1361-1373.

16. Geissmann, F., Jung, S., and Littman, D.R. 2003. Blood monocytes consist of two principal sub- sets with distinct migratory properties. Immunity. 19:71-82.

17. Ancuta, P., et al. 2003. Fractalkine preferentially mediates arrest and migration of CD16+ monocytes. J. Exp. Med. 197:1701-1707.

18. Sunderkotter, C., et al. 2004. Subpopulations of mouse blood monocytes differ in maturation stage and inflammatory response. J. Immunol. 172:4410-4417.

19. Qu, C., et al. 2004. Role of CCR8 and other chemokine pathways in the migration of monocytederived dendritic cells to lymph nodes. J. Exp. Med. 200:1231-1241.

20. Llodra, J., et al. 2004. Emigration of monocytederived cells from atherosclerotic lesions characterizes regressive, but not progressive, plaques. Proc. Natl. Acad. Sci. U. S. A. 101:11779-117784.

21. Zhao, L., et al. 2004. The 5-lipoxygenase pathway promotes pathogenesis of hyperlipidemia-dependent aortic aneurysm. Nat. Med. 10:966-973.

22. Lindquist, R.L., et al. 2004. Visualizing dendritic cell networks in vivo. Nat. Immunol. 5:1243-1250.

23. Randolph, G.J., Sanchez-Schmitz, G., Liebman, R.M., and Schakel, K. 2002. The CD16(+) (FcgammaRIII(+)) subset of human monocytes preferentially becomes migratory dendritic cells in a model tissue setting. J. Exp. Med. 196:517-527.

24. Tacke, F., et al. 2006. Immature monocytes acquire antigens from other cells in the bone marrow and present them to $\mathrm{T}$ cells after maturing in the periphery. J. Exp. Med. 203:583-594.

25. Germain, R.N., Miller, M.J., Dustin, M.L., and Nussenzweig, M.C. 2006. Dynamic imaging of the immune system: progress, pitfalls and promise. Nat. Rev. Immunol. 6:497-507.

26. Swirski, F.K., et al. 2006. Monocyte accumulation in mouse atherogenesis is progressive and proportional to extent of disease. Proc. Natl. Acad. Sci. U. S. A 103:10340-10345.

27. Jung, S., et al. 2000. Analysis of fractalkine receptor CX(3)CR1 function by targeted deletion and green fluorescent protein reporter gene insertion. $\mathrm{Mol}$. Cell. Biol. 20:4106-4114.

28. Niess, J.H., et al. 2005. CX3CR1-mediated dendritic cell access to the intestinal lumen and bacterial clearance. Science. 307:254-258.

29. Ginhoux, F., et al. 2006. Langerhans cells arise from monocytes in vivo. Nat. Immunol. 7:265-273.

30. Yates, R.M., and Russell, D.G. 2005. Phagosome maturation proceeds independently of stimulation of toll-like receptors 2 and 4. Immunity. 23:409-417.

31. Jung, S., et al. 2002. In vivo depletion of CD11c(+) dendritic cells abrogates priming of CD8 $(+) \mathrm{T}$ cells by exogenous cell-associated antigens. Immunity. 17:211-220.

32. Duffield, J.S., et al. 2005. Selective depletion of macrophages reveals distinct, opposing roles during liver injury and repair. J. Clin. Invest. 115:56-65. doi:10.1172/JCI200522675.

33. Van Rooijen, N., and Sanders, A. 1994. Liposome mediated depletion of macrophages: mechanism of action, preparation of liposomes and applications. J. Immunol. Methods. 174:83-93.

34. Nibbering, P.H., Leijh, P.C., and van Furth, R. 1987.
Quantitative immunocytochemical characterization of mononuclear phagocytes. II. Monocytes and tissue macrophages. Immunology. 62:171-176.

35. Smalley, D.M., and Ley, K. 2005. L-selectin: mechanisms and physiological significance of ectodomain cleavage. J. Cell. Mol. Med. 9:255-266.

36. Chereshnev, I., et al. 2003. Mouse model of heterotopic aortic arch transplantation. J. Surg. Res. 111:171-176.

37. Serbina, N.V., and Pamer, E.G. 2006. Monocyte emigration from bone marrow during bacterial infection requires signals mediated by chemokine receptor CCR2. Nat. Immunol. 7:311-317.

38. Weber, C., et al. 2000. Differential chemokine receptor expression and function in human monocyte subpopulations. J. Leukoc. Biol. 67:699-704.

39. Mack, M., et al. 2001. Expression and characterization of the chemokine receptors CCR2 and CCR5 in mice. J. Immunol. 166:4697-4704.

40. Cook, D.N., et al. 2001. Generation and analysis of mice lacking the chemokine fractalkine. Mol. Cell. Biol. 21:3159-3165.

41. Boring, L., et al. 1997. Impaired monocyte migration and reduced type 1 (Th1) cytokine responses in C-C chemokine receptor 2 knockout mice. J. Clin. Invest. 100:2552-2561.

42. Kuziel, W.A., et al. 1997. Severe reduction in leukocyte adhesion and monocyte extravasation in mice deficient in CC chemokine receptor 2. Proc. Natl. Acad. Sci. U. S. A. 94:12053-12058.

43. Kurihara, T., Warr, G., Loy, J., and Bravo, R. 1997. Defects in macrophage recruitment and host defense in mice lacking the CCR2 chemokine receptor. J. Exp. Med. 186:1757-1762.

44. Charo, I.F., and Taubman, M.B. 2004. Chemokines in the pathogenesis of vascular disease. Circ. Res. 95:858-866.

45. Daly, C., and Rollins, B.J. 2003. Monocyte chemoattractant protein-1 (CCL2) in inflammatory disease and adaptive immunity: therapeutic opportunities and controversies. Microcirculation. 10:247-257.

46. Lucas, A.D., et al. 2003. Smooth muscle cells in human atherosclerotic plaques express the fractalkine receptor CX3CR1 and undergo chemotaxis to the CX3C chemokine fractalkine (CX3CL1). Circulation. 108:2498-2504.

47. Green, S.R., et al. 2006. The CC chemokine MCP-1 stimulates surface expression of CX3CR1 and enhances the adhesion of monocytes to fractalkine/CX3CL1 via p38 MAPK. J. Immunol. 176:7412-7420

48. Wong, B.W., Wong, D., and McManus, B.M. 2002. Characterization of fractalkine (CX3CL1) and CX3CR1 in human coronary arteries with native atherosclerosis, diabetes mellitus, and transplant vascular disease. Cardiovasc. Pathol. 11:332-338.

49. Metlay, J.P., et al. 1990. The distinct leukocyte integrins of mouse spleen dendritic cells as identified with new hamster monoclonal antibodies. J. Exp. Med. 171:1753-1771.

50. Grisotto, M.G., et al. 2006. The human herpesvirus 8 chemokine receptor vGPCR triggers autonomous proliferation of endothelial cells. J. Clin. Invest. 116:1264-1273. doi:10.1172/JCI26666. 